\title{
El Cerdo Criollo Costero: Valioso recurso zoogenético local de la provincia de Buenos Aires Argentina
}

\author{
Carpinetti, B. '; Di Guirolamo, G. ${ }^{2}$; Delgado, J.V. ${ }^{3}$ y Martínez, R.D. ${ }^{2}$
}

\begin{abstract}
'Área de Gestión Ambiental y Ecología. Universidad Nacional Arturo Jauretche. Argentina.
${ }^{2}$ Facultad de Ciencias Agrarias. Universidad Nacional de Lomas de Zamora. Argentina.

${ }^{3}$ Facultad de Veterinaria. Universidad de Córdoba. España.
\end{abstract}

\section{PALABRAS CLAVE ADICIONALES}

Producción porcina .

Diversidad genética.

\section{RESUMEN}

El cerdo Criollo costero (CCC), forma parte de la cultura popular de la provincia de Buenos Aires. Se utiliza para producir carne y chacinados desde el principio de la colonización española hasta la actualidad. A pesar de esto, nunca se lo ha valorado zootécnicamente y no ha sido tenido en cuenta como recurso genético en la producción porcina moderna. El mayor número de ejemplares se mantiene como cerdo cimarrón en una amplia zona costera de la provincia. No se han estudiado aún sus características productivas ni su importancia económica y social. Se plantea aquí la necesidad de constituir un centro genético porcino local, destinado a caracterizar productivamente este recurso genético local para lograr un aprovechamiento más racional.

\section{Coastal Creole pig: Valuable local animal genetic resources in the province of Buenos Aires Argentina}

\section{SUMMARY}

\section{AdDITIONAL KEYWORDS}

Pig production.

Genetic diversity.

\section{INFORMACIÓN}

Cronología del artículo.

Recibido/received: 28.04.15

Aceptado/Accept: 27.01.16

Online: 15.09 .16

Correspondencia a los autores/Contact e-mail:

juanviagr218@gmail.com

\section{INTRODUCCIÓN}

Los cerdos domésticos ingresaron a América por primera vez a la Isla La Española, en el segundo viaje de Colón en 1493 y desde allí se expandieron hacia Colombia, Venezuela, Perú y Ecuador (Revidatti et al., 2004). A la Argentina llegaron con Pedro de Mendoza en la primera fundación de Buenos Aires en 1536 (Giberti, 1970). Luego hubo otras introducciones de cerdos y para fines del siglo XVI el número de animales era muy numeroso, tan es así que en la reunión del Cabildo de Buenos Aires efectuada el 16 de Junio de 1590, se acordó que los vecinos cumpliesen con la ordenanza de tener a los cerdos encerrados en un corral. Tal como ocurrió con las otras especies de interés zootécnico introducidas por los colonizadores españoles, de a poco fueron diferenciándo- se dos poblaciones, una mansa que vivía cerca de las ciudades en contacto con los humanos y otra de cría libre que habitualmente se denominó chancho cimarrón o feral. A mediados del siglo XVIII la población de cerdos cimarrones se había dispersado hacia el sur y llegaba hasta el actual partido de Balcarce, en la provincia de Buenos Aires. Según Cabrera y Yepes (1940), el sacerdote jesuita J. Cardiel describía en la época:

Hállase grande abundancia de puercos, que a cada paso se ven en grandes piaras o tropas, y mucho más si hay alguna laguna, o bañado inmediato. Susténtase de las raíces, que sacan hocicando la tierra. En cuanto al origen de estos puercos discurren variadamente los españoles. Dicen comúnmente que saldrían de las haciendas o estancias de Buenos Aires, donde años pasados no se hacía uso de su carne; y habiéndose 
por eso multiplicado, se metieron hacia el sud, llenando las campañas.

Ya en la segunda mitad del Siglo XIX, el célebre naturalista Guillermo Hudson (1949), describe en proximidades del estuario del Río de La Plata, las características de éstos cerdos:

Pertenecían a la cría de chanchos salvajes, descendientes originariamente del cerdo europeo, importado por los primeros colonos españoles y que, tras dos o tres siglos de vida agreste, habian cambiado bastante de la indole de sus progenitores. Este cerdo cimarrón, llamado barraco en la lengua del país, era un tercio más pequeño que el animal doméstico, con patas de mayor longitud y cabeza más puntiaguda y de un color uniforme rojo herrumbrado. Entre centenares no vi uno solo siquiera con manchas negras o blancas.

Transcurrieron casi 500 años desde la llegada de los primeros cerdos, pero la situación actual del cerdo Criollo (Linaje o raza formada a partir de estos planteles originales con la constante acción de la selección natural y adaptada a las condiciones ambientales particulares de la región), en la zona costera de la provincia de Buenos Aires es muy similar a la de la época colonial. Existe una importante población de cerdos Criollos en estado asilvestrado o cimarrón (figura 1), en el área de conservación de Bahía Samborombón. En ésta área natural protegida los cerdos silvestres son especialmente abundantes. Según diferentes estudios realizados sobre su situación poblacional a partir de censos aéreos, su número se habría incrementado significativamente en un período de 45 meses entre 1995 y 1998 (Merino y Carpinetti 2003) y de igual manera entre marzo del 2002, cuando se registraron 0,91 cerdos $/ \mathrm{km}^{2}$, y septiembre de

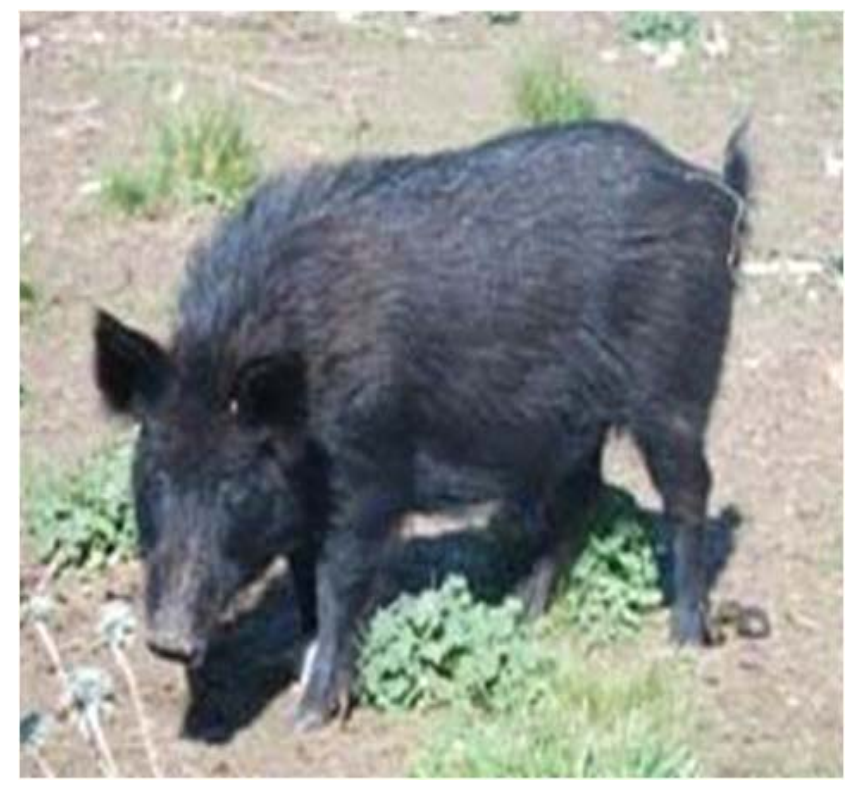

Figura 1. Cerdo Criollo Costero asilvestrado en la Bahía Samborombon. Denominado comúnmente Chancho $\mathrm{Ci}$ marrón (Coastal Creole feral pig in the Samborombon Bahía).
2005 con 7,78 cerdos $/ \mathrm{km}^{2}$ (Perez Caruzi et al., 2009). Existen además en el área costera unidades productivas extensivas y semiextensivas pequeñas y medianas que mantienen cerdos Criollos mansos para consumo familiar y fabricación de chacinados. El objetivo de éste trabajo fue describir y estudiar de manera preliminar la situación actual del cerdo Criollo costero (CCC), a efectos de lograr la concientización y sensibilización de los actores involucrados a efectos de constituir un centro genético porcino local, que permita generar conocimientos zootécnicos para un aprovechamiento racional de este recurso genético.

\section{MATERIAL Y MÉTODOS}

La Bahía de Samborombón (figura 2) es el humedal mixohalino más extenso de la Argentina (244 000 has), está ubicada en el noreste de la provincia de Buenos Aires, se extiende a lo largo de 150 kilómetros sobre la costa occidental del estuario del Río de la Plata, desde Punta Piedras ( $35^{\circ} 27^{\prime} \mathrm{S} ; 56^{\circ} 45^{\prime} \mathrm{O}$ ) hasta Punta Rasa $\left(36^{\circ}\right.$ $\left.22^{\prime} \mathrm{S} ; 56^{\circ} 35^{\prime} \mathrm{O}\right)$. Abarca en su extensión, una franja terrestre variable de 2 a $23 \mathrm{~km}$ de ancho y una porción de aguas someras hasta la isobata de 3,5 metros. Constituye una zona de interacción entre los ecosistemas acuático y terrestre, y de mezcla entre las aguas del Río de la Plata y el Océano Atlántico, lo que crea condiciones ecológicas particulares que le permite ser el sustento de una gran biodiversidad. Además de realizar una exten-

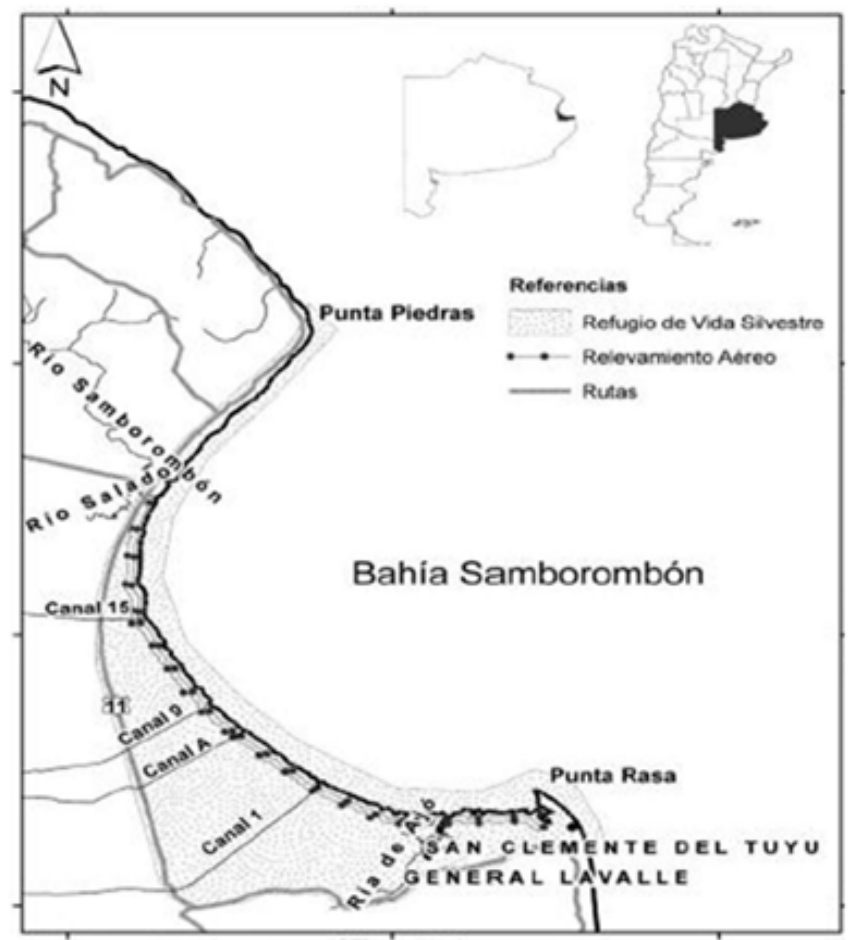

Figura 2. Zona Costera de la provincia de Buenos Aires donde habita el CCC (Coast zone of the province of Buenos Aires, inhabited by the CCC). 
sa e intensa búsqueda bibliográfica, se han realizado una serie de relevamientos de ésta zona costera de la provincia de Buenos Aires, tomando contacto con pequeños productores locales que utilizan cerdos Criollos para su producción de forma extensiva o semiextensiva con fines de consumo y comercial en pequeña escala. Además, en el contexto de un programa de vigilancia epidemiológica de enfermedades de los cerdos silvestres que dirige uno de los autores, se realizaron recorridas periódicas y capturas de individuos para pruebas serológicas, en la Reserva Natural Bahía Samborombón, dependiente de la Dirección de Aéreas Naturales Protegidas de la Provincia de Buenos Aires, acompañados por personal de guardaparques. Por último, hemos contactado con autoridades territoriales del municipio de Punta Indio a efectos de contar con su apoyo para la construcción de un centro genético porcino que nos permita generar conocimientos zootécnicos para un aprovechamiento racional de este recurso genético y su difusión entre los productores pequeños y medianos de la región.

\section{RESULTADOS}

Mediante la visita a la región se constató que el CCC es un recurso genético local, utilizado tradicionalmente por productores pequeños y medianos de la zona, en establecimientos de cría extensiva o semiextensiva. Generalmente los ejemplares los obtienen como producto de la caza de los cerdos cimarrones de la Bahía Samborombon, los que luego de un corto período de tiempo, se adaptan a su nueva situación de semicautiverio. Principalmente las crías y algunas hembras, se acostumbran rápidamente al contacto humano, mostrando mucha docilidad y mansedumbre que facilitan su manejo. Según los datos empíricos suministrados por las personas entrevistadas, las cerdas CCC, generalmente paren entre cuatro y ocho lechones, destetando generalmente el 100 por ciento de los mismos. Otra observación de interés es que tanto las cerdas como sus crías generalmente no se enferman y son muy tolerantes a distintas patologías. Por ejemplo, en granjas donde conviven con cerdas de razas mejoradas se observa claramente el mejor estado general de las cerdas CCC ante la presencia de sarna.

Otra característica favorable que observan los productores es que el CCC, está habituado al consumo de pastos, raíces y alimentos groseros, acostumbrándose rápidamente al consumo de distintos tipos de residuos o subproductos en general. Por otra parte también se observa que el volumen de alimento consumido por el CCC, es muy inferior al de las razas mejoradas, siendo ésta una condición fundamental para la subsistenciade los sistemas de producción familiares con mínima inversión.En general el producto final utilizado es el consumo del lechón, tanto en pureza (figura 3), como el producto de la cruza de la madre CCC con un padrillo

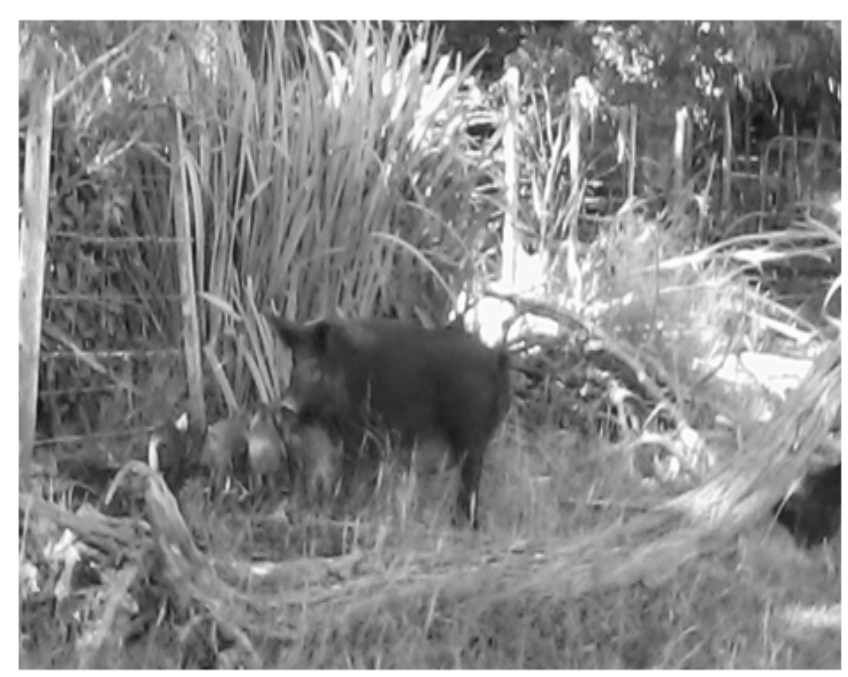

Figura 3. Cerda Criolla Costera en sistema de producción familiar con su primera camada. Las Tahonas Provincia de Buenos (Coastal Creole Sowin family production system with her first litter. The Tahonas. Province of Buenos Aires).

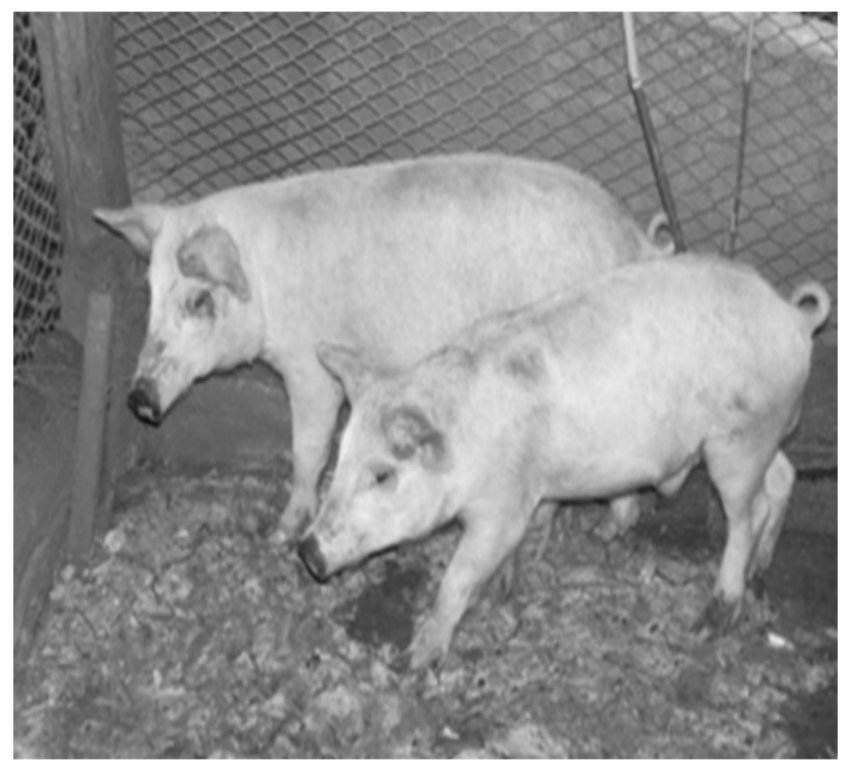

Figura 4. Lechones F1 en recría (CCC x Landrace). Verónica. Provincia de Buenos Aires (F1 in rebreeding piglets (CCC $x$ Landrace). Veronica. Province of Buenos Aires).

(figura 4) perteneciente a una raza mejorada (generalmente Landrace, Yorkshire o Duroc Jersey). Oros productos generados, son los relacionados con la fabricación de distintos tipos de chacinados (jamones, chorizos etc.), que se caracterizan por su sabor particular y su bajo contenido graso, estos se hacen a partir de cerdos de 80 - $100 \mathrm{~kg}$. de peso vivo, luego de concluir su etapa de engorde (figura 5).

\section{DISCUSIÓN}

La necesidad de caracterizar las razas Criollas de cerdos está dada por su importancia como recurso 


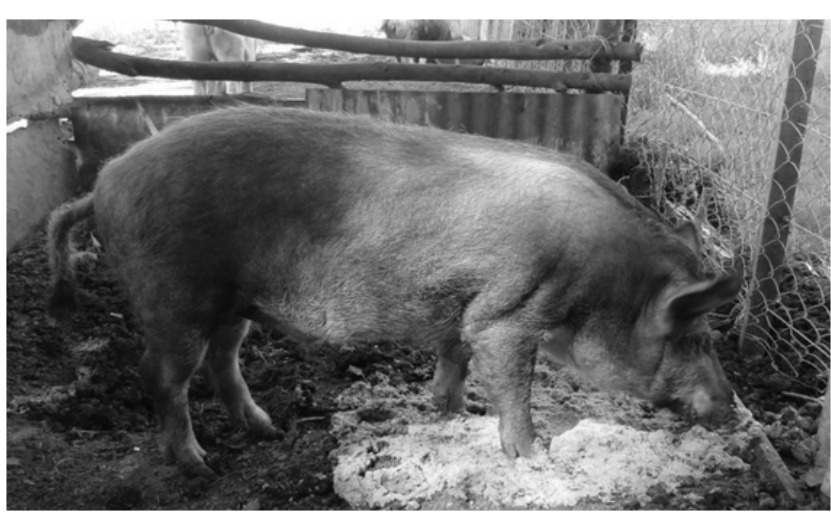

Figura 5. Capón Cerdo Criollo Costero en la etapa de engorde. Las Tahonas Provincia de Buenos (Coastal Creole pig fattening. The Tahonas. Province of Buenos Aires).

genético con potencial inexplorado, sobre todo para la generación de chacinados regionales. Un buen ejemplo es el emblemático resurgimiento del cerdo Ibérico para la producción de embutidos en España, y que estaba desapareciendo de forma acelerada debido al uso de razas comerciales modernas por parte de la mayoría de los productores porcinos (Amurrio, 1996; Castro, 2000). Según algunos autores, la población porcina de cerdos Criollos en Latinoamérica hacia la década de 1970 era del $80 \%$ mientras que hoy se habría reducido sensiblemente ante el avance de otras razas (García et al., 2008; Linares et al., 2011; Pérez Carusi et al., 1999). Tal como sugiere Revidatti (2005), estos linajes de cerdos están representados por animales contenidos en poblaciones ferales o relacionados a pequeñas explotaciones familiares de subsistencia, lo cual es concordante con el hecho de que dichos productores no tienen el acceso a la compra de material genético de pedigree (McManus et al., 2010). En este trabajo, se ha constatado que el cerdo Criollo costero CCC es un recurso genético local que forma parte de la cultura popular bonaerense, ya que según los testimonios recabados se lo ha utilizado de manera productiva desde el principio de la colonización española y hasta la actualidad. Paradójicamente, nunca se le ha reconocido su valor zootécnico ni sus cualidades productivas. Aunque la introducción de razas mejoradas se inició en 1900 (Carrazzoni, 1997), hace más de cien años, los productores lo siguen utilizando y prefiriendo en muchos casos. Por otra parte, trabajos recientes realizados a partir del marcador molecular mitocondrial citocromo b, confirman la asociación del linaje de cerdos Criollos costeros de la Bahía de Samborombón a las razas Ibéricas (Figueroa, 2014). Situaciones similares a la descripta para el CCC han ocurrido en distintas zonas de Latinoamérica, donde se ha avanzado sustancialmente en el conocimiento zootécnico y en la utilización productiva racional del cerdo Criollo, por poner algunos ejemplos podemos citar al cerdo Pampa Rocha del Uruguay (Vadell, 2008), a distintas variedades descriptas en Ecuador como el Zungo, el Casco de Mula, el Congo Santandereano, el
Pelón y el Cuino (Escobar Ribera, 2007), al cerdo Criollo de la región Cho'rti' del departamento de Chiquimula en Guatemala (Lorenzo et al., 2012) y al cerdo Criollo del Estado de Apure en Venezuela (Hurtado et al., 2005). La formación de un centro genético porcino local, orientado a caracterizar zootécnicamente al CCC, a valorizar sus productos y a producir reproductores evaluados genéticamente y sanitariamente contribuirá al desarrollo económico y social de la costa bonaerense.

\section{BIBLIOGRAFÍA}

Amurrio M.F. 1996. Caracterización del cerdo Criollo del valle de Tipajara en la Provincia de Misque. Tesis de ingeniero Agrónomo. Facultad de ciencias Agrícolas, Pecuarias, Forestales y Veterinarias. Universidad Mayor de San Simón, Cochabamba, Bolivia.

Cabrera, A. y Yepes, J. 1940. Mamíferos sudamericanos. Vida, costumbres y descripción. Compañía Argentina de Editores. Buenos Aires.

Carrazzoni, J.A. 1997. Crónicas del campo argentino (Nuestras raíces agropecuarias). Serie de la Academia Nacional de Agronomía y Veterinaria. $N^{\circ}$ 23. 403 pp.

Castro, S., Albuquerque, M. and Germano, J.L. 2000. Conservation of local pigs in Brazil. Proc. V Global Conference in Conservation of Domestic Animal Genetic Resources. Embrapa Recursos Genéticos e Biotecnologia. Brasília. Brasil. CD-ROM. 3 pp.

Escobar Ribera, J.C. 2007. Caracterización y sistemas de producción de los cerdos Criollos del Cantón Chambó. Tesis de grado. Escuela Superior Politécnica de Chimborazo. Facultad de Ciencias Pecuarias. $119 \mathrm{pp}$.

Figueroa, C. 2014. Variabilidad genética en los planteles de los pequeños productores porcinos del Noroeste de la provincia de Buenos Aires. Trabajo de grado de la Licenciatura en Genética. Escuela de Ciencias Agrarias, Naturales y Ambientales. Universidad Nacional del Noroeste. Buenos Aires.

García, G.; Santana, I.; Rico, C., Pérez, E.; Ly, J.; Diéguez, F.J.; Agüero, L.; García, A.; Roque, R.; Velázquez, F. y Tosar, M. 2008. Conservación, evaluación, mejora y uso del cerdo Criollo Cubano. Rev Comp Prod Porc, 15: 85-89.

Giberti, H. 1970. Historia económica de la ganadería Argentina. Editorial Solar/Hachete. Buenos Aires. 217 pp.

Hudson, G.E. 1949. Allá lejos y hace tiempo. Ediciones Peuser. Buenos Aires.

Hurtado, E.; González, C. y Vecchionacce, H. 2005. Estudio morfológico del cerdo Criollo del estado Apure, Venezuela. Rev Zootec Trop, 23: 17-26.

Linares, V.; Linares, L. y Mendoza, G. 2011. Caracterización etnozootécnica y potencial carnicero de Sus scrofa cerdo Criollo en Latinoamérica. Sci Agropec, 2: 97-110.

Lorenzo, M.; Jáuregui, J. y Vásquez, C.H. 2012. Caracterización del cerdo Criollo de la región Cho'rti' del departamento de Chiquimula, Guatemala. Revista AICA, 2: 103-108.

McManus, C.; Rezende Paiva, S.; Rezende Silva, A.V., Sayori Murata, L.; Louvandini, H.; Barrera Cubillos, G.P.; Castro, G.; Martínez, R.A.; Llambi Della casa, M.S. and Pérez, J.E. 2010. Phenotypic characterization of naturalized swine breeds in Brazil, Uruguay and Colombia. Braz Arch Biol Technol, 53: 583-591.

Merino, M.L. and Carpinetti, B. 2003. Feral pig Sus scrofa population estimates in Bahía Samborombón conservation area, Buenos Aires Province, Argentina. Mastozoología Neotropical. J Neotrop Mammal, 10: 269-275. 
Pérez Carusi, L.C.; Beade, M.S.; Miñarro, F.; Vila, A.R.; GiménezDixon, M. and Bilenca, D.N. 2009. Relaciones espaciales y numéricas entre venados de las pampas (Ozotoceros bezo articus celer) y chanchos cimarrones (Sus scrofa) en el Refugio de Vida Silvestre Bahía Samborombón, Argentina. Ecol Austral, 19: 6371.

Revidatti, M.A.; Capellari, A. y Martínez, R. 2004. Recursos genéticos porcinos en Argentina. In: J.V. Delgado Bermejo (Ed.).
Biodiversidad porcina iberoamericana. Universidad de Córdoba. España. pp. 111-133.

Revidatti, M.A.; Capellari, A.; Prieto, P.N. y Delgado, J.V. 2005. Recurso genético porcino autóctono en el Nordeste de la República Argentina. Arch Zootec, 54: 97-100.

Vadell, A. 2008. Una reseña corta sobre la raza Criolla de cerdos Pampa Rocha y su utilización en Uruguay. Rev Comp Prod Porc, 15: 105-112. 